\title{
Case study of muscle fatigue in parafunctional patient using occlusal device with fiber Bragg grating sensors
}

\author{
Paula F. Nascimento ${ }^{1}$, Ana P. G. O. Franco ${ }^{1}$, Rodrigo Fiorin ${ }^{1}$, Mauren A. de Souza ${ }^{2}$, \\ Hypolito J. Kalinowski ${ }^{3}$, Ilda Abe ${ }^{1}$ \\ ${ }^{1}$ Federal University of Technology - Paraná, 80230-901 Curitiba, Brazil \\ ${ }^{2}$ Pontifical Catholic University of Paraná, 80215-901 Curitiba, Brazil \\ ${ }^{3}$ Universidade Federal Fluminense, 24210-240 Niterói, Brazil \\ e-mail: ildaabe@utfpr.edu.br
}

\begin{abstract}
This case study describes a new method using a fiber optic Bragg grating sensor to determine muscle fatigue in a patient wearing a resilient occlusal device for the treatment of bruxism symptoms. Four tasks performed at maximum bite force induced rapid exhaustion for the analysis of fatigue. The results show that it is possible to obtain a better understanding of the muscle fatigue process by using the fiber sensor. Differences between the bite patterns are detected at each phase of the induced fatigue. It is possible to compare the bite peak sensitivity coefficients for the exhaustion phase, $1.37 \mathrm{Ns}^{-1}$, and for fatigue phase, $2.54 \mathrm{Ns}^{-1}$. Additionally, the increase in the facial thermal profile is correlated to the different bite force pattern performed.
\end{abstract}

Index Terms — bruxism, muscle fatigue, occlusal device, fiber Bragg grating.

\section{INTRODUCTION}

Bruxism is a motor disorder of sleep characterized by increased muscular activity of the masseter and temporalis muscles, thus causing grinding and abrasion of dental tissues [1]. Bruxism is a repetitive and recurrent episode of rhythmic masticatory muscle activity [2], [3]. These episodes are observed in $60 \%$ of the general adult population during sleep [4], [5].

Temporomandibular disorders (TMD) are defined as a complex, chronic and recurrent painful musculoskeletal disorder that involves the temporomandibular joint and associated structures, such as the soft tissues around it [6], [1]. The etiology of TMD is multifactorial and it is probably due to muscular hyperfunction, parafunctional habits, traumatic injury, hormonal influence or internal joint derangement [7]. Emotional stress and muscle tension contribute and intensify the TMD, becoming more difficult its recovery [8].

The myofascial pain syndrome is clinically detected in patients with TMD during the jaw movements and palpation. This evidence is related to muscle hyperactivity and alterations in the muscle motor behavior during chewing. Symptomatic TMD patients have large amplitude of muscle activity, lower functional efficiency and predisposition to muscle fatigue [9].

The muscle fatigue is characterized by the inability of the muscles to generate high levels of 
muscular strength or to maintain current levels over a longer period of time [10], [11]. The effects of fatigue can be observed by the decrease of its contraction speed and by the increase of the relaxation time after the maximal and submaximal exercises [12]. The muscular voluntary activity starts with processes controlled by the brain and it ends with the formation of cross-bridges within the muscle fibers.

The muscle fatigue can occur by failure of any of the processes responsible by muscular contraction [13]. Furthermore, it is identified as a defense mechanism against harmful effects on the integrity of the muscle fibers [14]. The fatigue is commonly observed in endurance sports and daily activities [15]. It can be classified into central - mainly due to a disorder of neuromuscular transmission between the central nervous system and the muscular membrane; or peripheral - where there is a modification in the involved muscles. The muscle fatigue depends on the type and intensity of exercises, the type of the muscular fibers being recruited, the level of subject training, and the environmental conditions [16]. Muscle fatigue is also related to an increase in the metabolites concentration and changes in both speed of conduction of the muscle fibers and the number of recruited motor units [17]. Additionally, it is related as a predisposing factor of headaches and facial muscle pain presented by the patients. Farella et al. [18] reported that the pain of the masticatory muscles after clenching depends on the level of force exerted by the patient. As the temperature increases, blood flow and muscle injury are among the causal factors of muscle fatigue. For this reason, muscle fatigue is also investigated by thermal facial changes (facial thermography analysis) within the main muscles involved during chewing [19], [20].

The muscular hyperfunction, the prolonged isometric contraction (e.g., clenching), and functional unbalances (e.g., overload during chewing) increase the blood flow, with consequent reduced supply of oxygen to the muscle and rapid accumulation of metabolites that interfere with the muscle contractile function, inducing local ischemia and hyperemia during and/or after contraction. Consequently, there is an increase of the surface temperature on the skin and it may produce the feeling of fatigue in these muscles [21], [22].

There are reports of electromyographic studies performed for the investigation of muscle fatigue in vivo [11], [12]. These studies have simulated muscle fatigue in the isometric conditions. In general, during the isometric movement occurs an increase in electromyographic signal components in the time domain; on the other hand, at the frequency domain, it happens a reduced signal at lower frequency regions. During the submaximal exercise, the response of the muscle to fatigue is translated into an increased number of the motor units recruited, and/or in its synchronization. This behavior is intended to compensate for the reduced ability to generate force by motor units. This response is observed by increasing the amplitude of the electromyographic signals [13].

Fiber Bragg Gratings (FBG) are intrinsic sensors that can be recorded in the core of optical fibers and have many advantages in biomechanical and biomedical researches when compared to other sensing technologies [23]. The FBG have small dimensions and have temperature and pressure Brazilian Microwave and Optoelectronics Society-SBMO received 01 Apr 2018; for review 05 Apr 2018; accepted 11 June 2018 
capability compatible with human physiology, the fiber optic is flexible, resistant to the majority of corrosive environments, non-electric and non-conductive device, immune to electromagnetic noise and radio frequency interference, resistant to moisture and chemicals present during in vivo measurements. The optical fiber is made of silicate glass and therefore biocompatible.

Biomechanical measurements with FBG sensors have been reported in the literature [24]-[30] for applications of both in vitro and in vivo conditions. FBGs also have been used to monitor orthodontic forces [24]-[29], [31], [32]. Tjin et al. demonstrated the monitoring of pressure and temperature for a dental device as a function of time for apnea patients [29]. The tactile sensor arrays using fiber Bragg grating sensors was reported by Heo et al. who had used an axle bridge in order to avoid the nonuniform strain distribution [32]. Forces at several teeth as a function of the load applied has been evaluated by Milczewski et al. by using special fibers, high birefringence fiber or polymeric fiber [26], [28]. FBG sensors were also used for monitoring forces on the internal parts of the teeth within an artificial maxilla model by Milczewski et al. [27]. The use of FBG for measuring different jaw movements, performed for patients with occlusal parafunctional by using occlusal devices, was demonstrated by Franco et al. [25]. Abe et al. reported the use of FBG sensors applied in the measurement of the magnitude of a human bite in the molar intercuspidation [24]. More recently, Umesh et al. demonstrated a transducer formed by a FBG and a box structure $(10 \times 10 \times 10 \mathrm{~mm})$ of aluminum for measurement of the bite force [31].

There are a limited number of reports in the literature about muscle fatigue during the use of resilient occlusal devices. Therefore, there is a need for studying muscle fatigue in patients who are wearing resilient occlusal devices for the treatment of bruxism symptoms.

In this research, we demonstrated the use of FBG sensors to characterize the process of inducing and monitoring muscular fatigue. The FBG sensor was inserted in a resilient occlusal device, previously characterized (in vitro), which was used by a parafunctional patient for in vivo analysis. The characterization of fatigue was obtained by inducing muscle exhaustion. The fatigue can be associated with the increase of muscular activity. These changes can be associated with the variations of temperature; therefore the infrared thermography images were also acquired.

\section{MATERIAL AND METHODS}

FBG were inscribed in G.652 telecommunications fiber using an Excimer $\mathrm{KrF}$ laser (Coherent, Xantos XS 500) at $248 \mathrm{~nm}$, whose beam illuminates the fiber through a phase mask. The fiber samples were hydrogen loaded $\left(100 \mathrm{~atm}, 25^{\circ} \mathrm{C}, 4\right.$ days) before the grating production, in order to increase its photosensitivity to the UV-light. The laser was operated at $5 \mathrm{~mJ}$ per pulse with $200 \mathrm{~Hz}$ repetition rate. The estimated length of the gratings is approximately $3 \mathrm{~mm}$, derived from the laser beam diameter after the iris. The acquisitions of the optical spectra were performed during the writing 
process of the gratings and along the experiments by using a FBG interrogator (Micron Optics, SM125-500).

In order to make the resilient occlusal device to in vivo and in vitro tests, the impressions of the upper and lower arches were performed with alginate (Cavex Color Change Fast Set Dustfree, Haarlem). The gypsum model (Durone, Dentsply) of the arches were obtained from this impression. The occlusal contact of the upper left first molar was marked with carbon paper into the mouth of the patient.

To perform the in vitro test, the same gypsum model was accomplished in a semi-adjustable articulator (4000-S Articulator, Bioart), at the occluded position. The semi-adjustable articulator is a device that records the maxillo-mandibular relations and intends to reproduce the jaw movements. This equipment allows the fixation of gypsum models that reproduce the arcades of the patient for observation and analysis of the maxilla mandibular movement without the presence of the patient. The articulator was adjusted using mean standard values of the mandible: horizontal condylar inclination in the glenoid fossa of $30^{\circ}$ and Bennett side shift of $15^{\circ}$, at the balancing side with the sagittal plane during the lateral movement of the mandible. The condylar and Bennett angles are used to reproduce the human physiological condition.

The gypsum model was positioned into a vacuum plasticizing (Vacuum Plasticizer Nite White, Nite White), where a silicone plate (Whiteness FGM, Dentscare Ltda) was heated and pressed on the model. The FBG sensor was positioned and fixed with cyanoacrylate on the stronger occlusal contact point of the upper left first molar. After this, another silicon plate was heated and pressed on the assembly. The final thickness of the occlusal device is of $2 \mathrm{~mm}$. Fig. 1(a) shows the gypsum model, the occlusal device and Fig. 1(b) shows the assembled system used for the in vitro test. After the in vitro tests, the same occlusal device with the FBG embedded sensor was used for in vivo experiments.

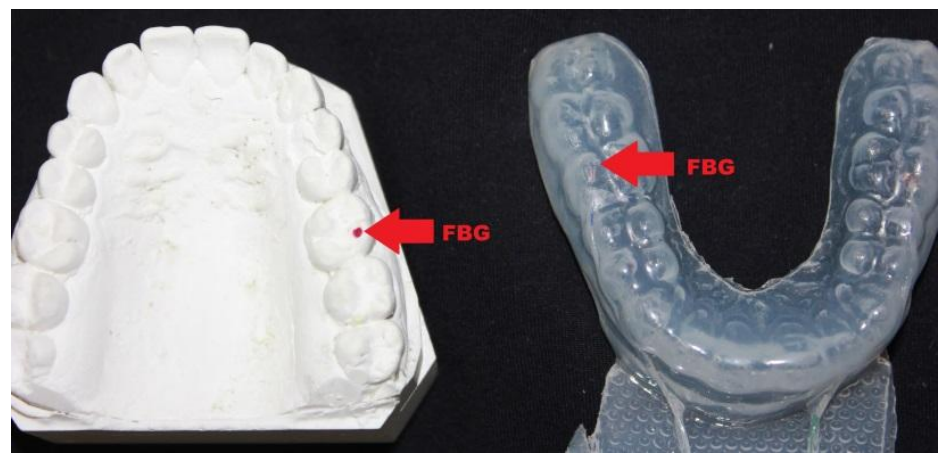

(a)

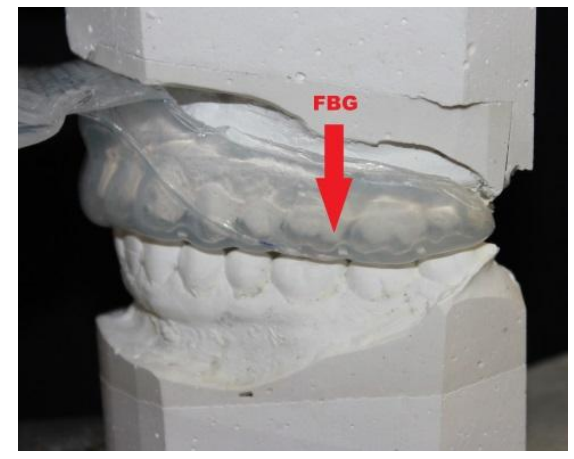

(b)

Fig. 1.(a) Gypsum model of upper arch showing the contact point of upper first molar, and the resilient occlusal device with the embedded sensor; (b) the gypsum model in the occluding position with the occlusal device and the FBG sensor placed in the left first molar. 


\subsection{In vitro analysis}

The in vitro study was performed using a mechanical system with a lever and calibration weights. Loads ranging from 0 to $40 \mathrm{~N}$ were applied over the left side at the occluded gypsum model.

It is important to notice that the occlusal plane of the gypsum model is not perpendicular to the applied load direction; therefore there are components of the applied force along the two perpendicular directions on the occlusal plane, i.e., longitudinal and transversal forces at the occlusal plane. The longitudinal forces induce longitudinal strains at the FBG sensor and it is possible to analyze changes in the load by the shift of the spectral reflection band of the sensor. Higher load forces applied to the system can also induce birefringence at the fiber, due to the transversal components of the force. However, the longitudinal strain is the principal involved component in this work.

The displacement of the spectral position of the grating as a function of the applied load is acquired for the in vitro analysis. The sensitivity coefficient, $\mathrm{d} \lambda / d F$, is obtained from the best fit to the data. Once the sensitivity of the sensor is obtained, using the wavelength shift and the coefficient of sensitivity, it is possible to obtain the associated force. Equation 1 relates the changes of the force with respect to the observed spectral shift of the wavelength.

$$
\Delta F=\left(\frac{d \lambda}{d F}\right)^{-1} \Delta \lambda
$$

\subsection{In vivo analysis}

The in vivo analysis was performed at a female patient with bruxism. Fig. 2(a) shows the patient with the occlusal device inside her mouth. In Fig. 2(b) it is possible to see a closer detail with the embedded optical fiber inside the device, which is located at the first molar. The FBG sensor is responsible for monitoring the deformations that occurred at the upper left first molar during the different movement tasks, which were performed in order to identify and characterize the muscle fatigue. The experiments were accomplished in a controlled environment temperature $\left(21^{\circ} \mathrm{C}\right)$.

When the device is positioned into the mouth of the patient, there is an initial shift in the spectral position of the grating resonance band due to the increase of temperature, from room temperature to internal body temperature. A waiting interval was used, until the thermo-stability of the sensor was achieved; subsequently the patient was asked to perform different movements.

The movements were divided into four tasks:

I. Three maximum bites with duration of $15 \mathrm{~s}$ and relaxation of $180 \mathrm{~s}$;

II. Three maximum bites with duration of $15 \mathrm{~s}$ and relaxation of $60 \mathrm{~s}$;

III. Sequential maximum bites with duration of $20 \mathrm{~s}$ and relaxation of $5 \mathrm{~s}$ until muscle exhaustion and presence of pain; 
IV. Sequential maximum bites with duration of $10 \mathrm{~s}$ and relaxation of $3 \mathrm{~s}$ until muscle exhaustion and presence of pain.

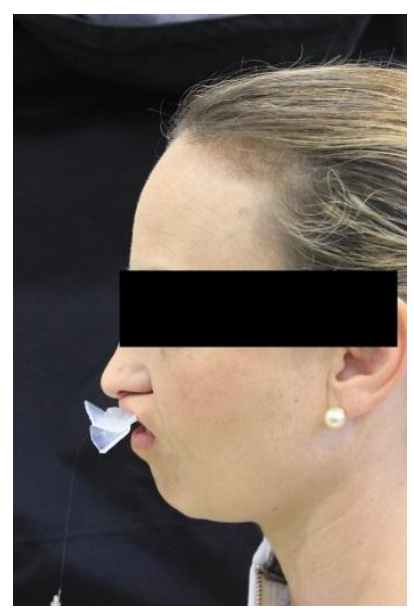

(a)

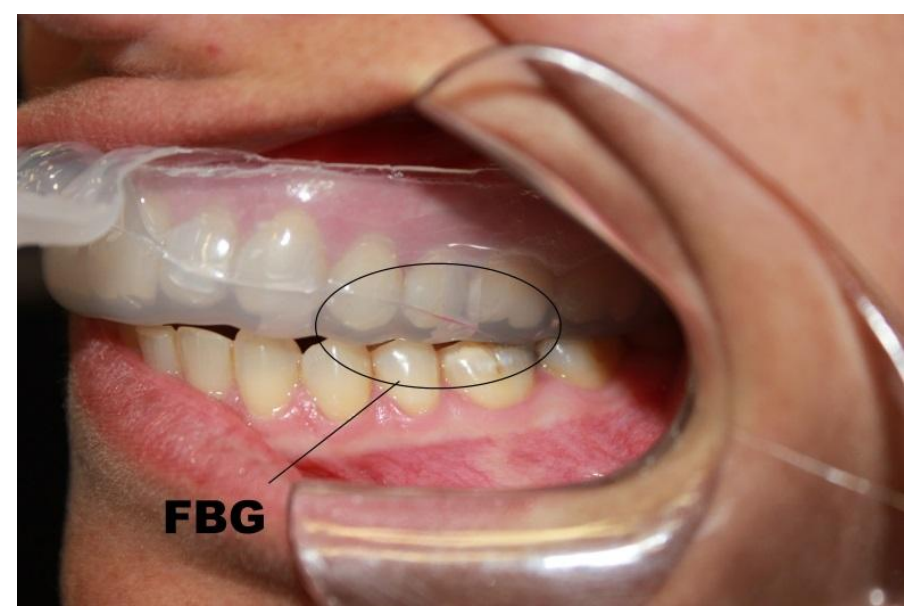

(b)

Fig. 2. (a) Photograph of the patient wearing the occlusal devicewith the embedded FBG sensor; (b) Detail of the occlusion device at bite position.

\subsection{Thermal analysis}

In order to verify facial and masticatory muscle temperature changes, infrared images were collected from the face. The images were collected before the beginning of the tasks, after each movement and $5 \mathrm{~min}$ after the end of the experiment. An infrared camera registered the thermal radiation and produced thermal images of the face surface in which each pixel corresponds to a temperature value. Regarding this thermal image acquisition, it was employed a camera (A325, FLIR) with a resolution of 320 by 240 pixels, corresponding to a temperature range from -20 to $120^{\circ} \mathrm{C}$, thermal resolution of $0.05^{\circ} \mathrm{C}$. This camera enables 30 frames/second images for video recording. For the thermal analysis, it was employed a FLIR software (ThermaCam Researcher Pro 2.9). A rotatory system was installed inside an acclimatized room, in which temperature and humidity are controlled, avoiding any undesired airflow. The infrared camera is fixed onto that rotatory support, which turns around the volunteer, allowing the acquisition of patient's temperature changes during the masticatory process.

\section{RESULTS\& DISCUSSION}

\subsection{In vitro analysis}

Initial tests of bite forces were performed in the static occlusion model, in order to obtain the sensitivity of the sensor. Several spectra of FBG were obtained for different loads, which were applied over the left side of the model.

Fig. 3 shows the curve that describes the shift of the FBG spectral position as a function of the applied load at the left side of the gypsum model, with the sensor positioned also at the left side. The sensitivity coefficient was $0.028 \mathrm{nmN}^{-1}$ for the sensor located at the upper left first molar. The 
sensitivity value was obtained from the best linear fit for the data (correlation coefficient 0.997). From the obtained slope it is possible to determine the force as a function of the applied load along the tasks.

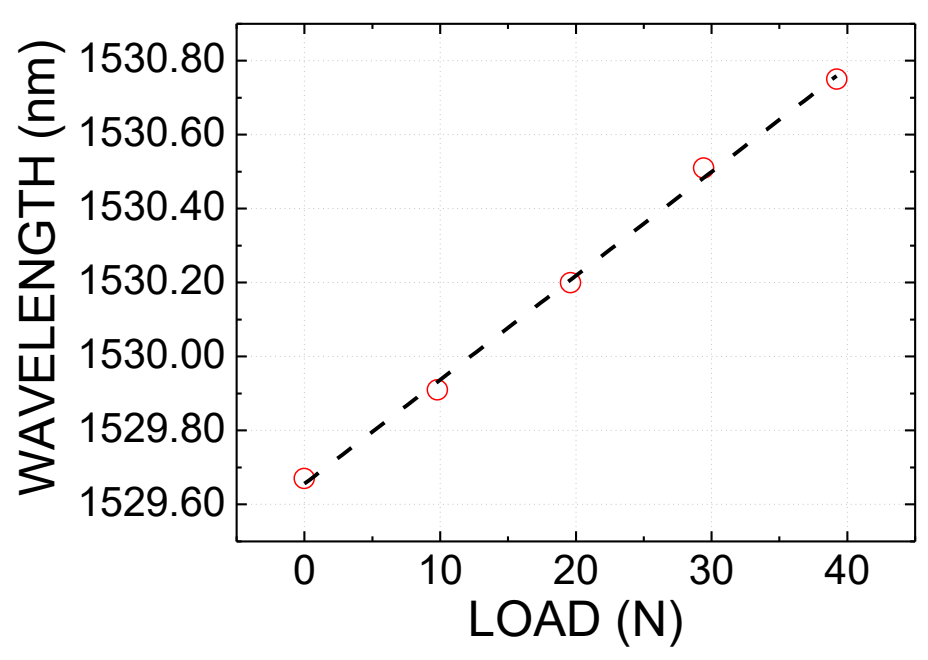

Fig. 3. Position of the FBG resonance band as function of the loads applied at the upper left first molar. (Symbols represent the experimental data; the dashed line is the linear best fit).

\subsection{In vivo analysis}

When the device is positioned into the mouth of the patient, there is an initial shift in the spectral position of the grating resonance band due to the increase of temperature, from room temperature to internal oral cavity temperature. A waiting interval of approximately 2 min was used, until the sensor achieved thermo-stability (see Fig. 4).

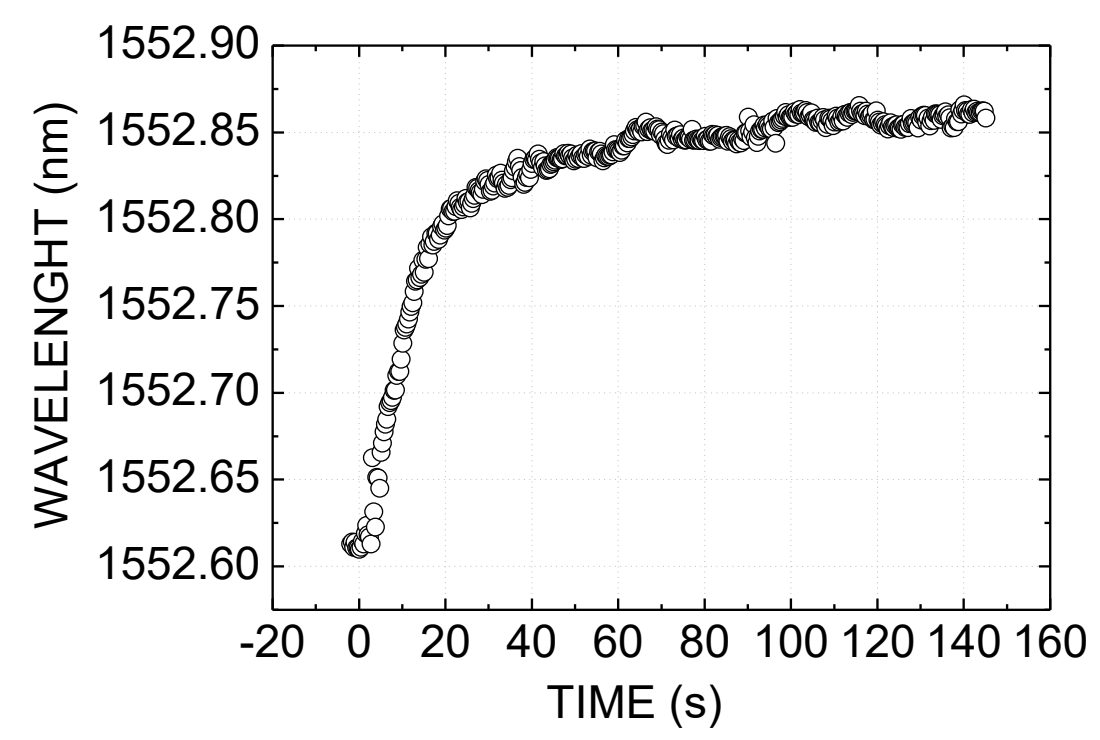

Fig. 4. Evolution of the spectral position of the sensor inside the mouth of the patient, due to the internal temperature. (Symbols are the experimental data). 
The graphs on Fig. 5(a) and 5(b) show results from experiments conducted with a low frequency rate (sampling) in order to obtain the standard behavior of the bite force amplitude (bite cycle). The two experiments were performed with bites of short period $(15 \mathrm{~s})$; changing only the relaxation period: for the first time the relaxation period was of $180 \mathrm{~s}$ (task I) and the second time, it was performed with a minor interval (60 s- task II). The obtained results verify the repeatability of the system. It is possible to observe that after each bite cycle, approximately $40 \mathrm{~N}$ (bite force amplitude) for both tasks, the curve returns to the same initial value.

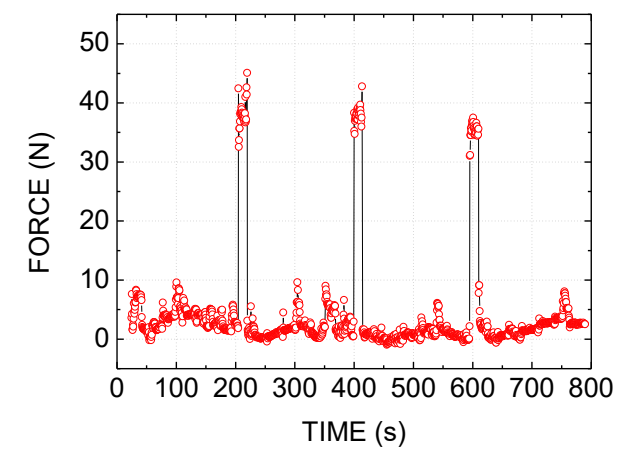

(a)

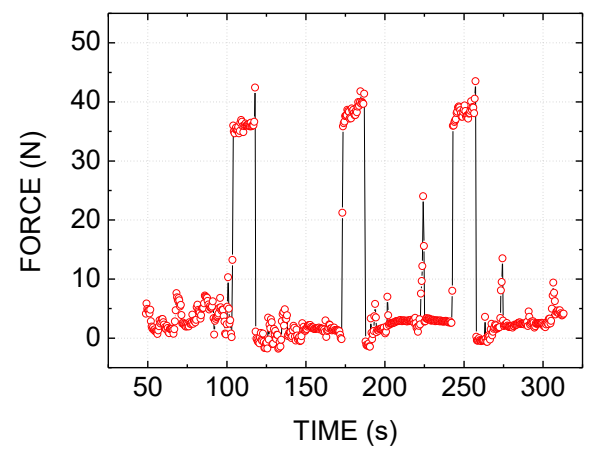

(b)

Fig. 5. Maximum bite force amplitude (bite cycle) in a situation of low repetition rate and short time of bite: (a) Task I and (b) Task II. (Symbols are the experimental data; line is only a guide to the eyes).

The graphs on Fig. 6(a) display results obtained with a longer maximum bite time (of $20 \mathrm{~s}$ ), which was used (isometric movement) in the attempt of inducing fatigue more quickly (task III). Therefore, exhaustion was used for stimulation of fatigue. In this graph, it can be observed that the first three bite cycles have appropriate repeatability. However, after that (at the fourth bite cycle), it occurs a more evident reduction in the bite force amplitude as a function of the time that is followed by an increase in muscle activity, where other motor units are recruited to compensate the fatigue of the main muscle group. The graphs on Fig. 6(b) show in detail the last two bite cycles (fourth and fifth in the graphs of Fig. 6(a)), and it is possible to notice that the bite force over time is not constant but increases steadily from the start of the bite action (this trend is also partially perceptible in the third bite cycle in the graphs of Fig. 6(a)). Using a linear best fit procedure for the bite force along time, it is possible to determine the slopes of $0.73 \mathrm{Ns}^{-1}$ and $1.28 \mathrm{Ns}^{-1}$ for the fourth and fifth bite cycles, respectively. The longer time needed to reach the maximum bite force may indicate that the fatigue process took place, which is also supported by the higher amplitude value in the fifth bite cycle, resulting from the additional muscles involved. 


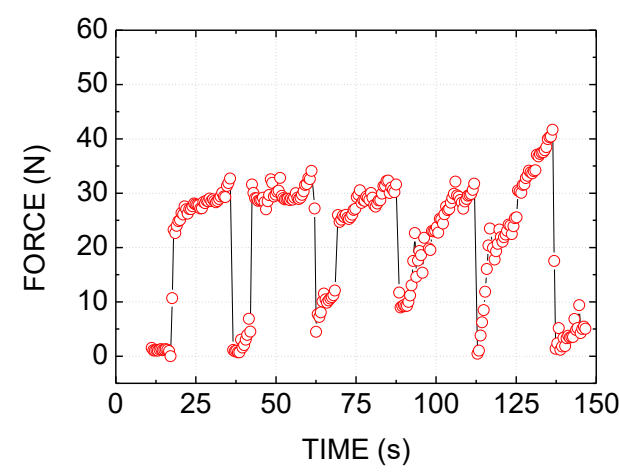

(a)

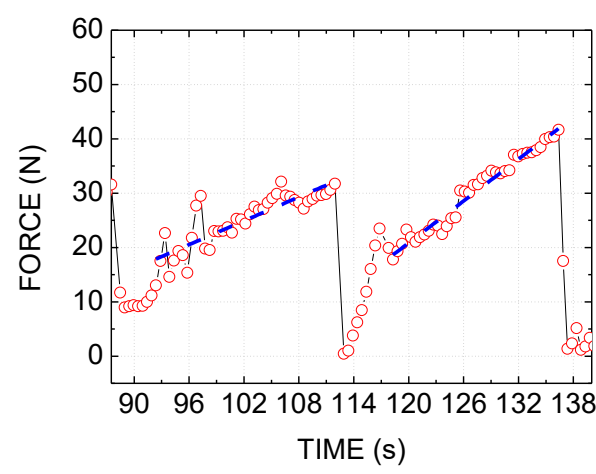

(b)

Fig. 6. (a) Task III: Higher maximum bite time used in order to induce exhaustion; (b) Detail of last two bite cycles showing the increase of the bite force along with the time. (Symbols are the experimental data; line is only a guide to the eyes; dashed line is the linear best fit).

The evolution of the bite force along three different phases (based on the relaxation time of $3 \mathrm{~s}$ - task IV) is presented on the graphs of Fig. 7(a). It is possible to notice that each phase presents a characteristic pattern for each bite cycle. The first six bite cycles correspond to the first phase, identified by the characteristic of repeatability; to induce exhaustion a higher maximum bite time (10 s) was used, it can be perceived that the maximum bite force is constant along the closure. The seventh bite cycle shows the start of the fatigue process as described in the previous paragraph. The eighth to twelfth bite cycles are characterized as the second phase, where it can be observed a reduction in the initial bite force, which is followed by an increase of the force along the time (as also discussed at the end of the previous paragraph).

The maximum force at each bite cycle is higher as compared to those of the bite cycles in the first phase. This behavior was also observed in the last bite cycles of task III. This means that other motor units are being recruited in order to compensate for the reduction of the maximum bite force caused by exhaustion. Accordingly, from the thirteenth to eighteenth bite cycles (third phase); it can be observed that the initial maximum force is reduced and the maximum force amplitude value is lower if compared to the second phase.

This behavior suggests that, from this moment on, the recruited motor units cannot maintain the maximum bite force due to the presence of muscular pain. This behavior is identified as muscle fatigue. The graphs on Fig. 7(b) show the comparison of the last bite peak of second phase and the first bite of the third phase. The dotted lines show the best fit of the bite force along the time. The obtained slope for the second phase has a value of $1.37 \mathrm{Ns}^{-1}$, whereas the slope in third phase presents a greater value, $2.54 \mathrm{Ns}^{-1}$. Another perceived difference is that the maximum bite force attained, which is almost $20 \%$ lower in the third phase, as compared to the second phase. This means that the third phase can be associated to higher fatigue process (point of fatigue) [10]. During these tasks, it had not been detected significant temperature changes, despite of the initial thermo-stability. 


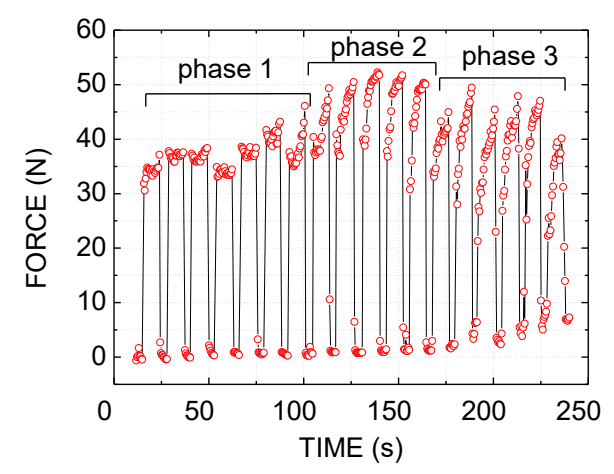

(a)

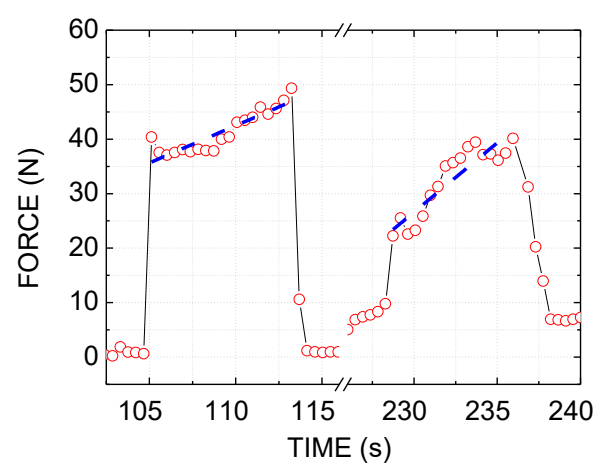

(b)

Fig. 7. (a) Phases of fatigue induction (task IV): higher maximum bite time (phase 1), exhaustion process (phase 2) and point of fatigue (phase 3); (b) Detail of the bite cycles for the second and third phase, showing the increase of the bite force along with time. (Symbols are the experimental data; line is only a guide to the eyes; dashed line is the linear best fit).

\subsection{Thermal analysis}

In order to investigate the changes in the temperature, infrared thermography images were collected, and a preliminary thermal analysis was performed to study the muscles involved during chewing. Infrared thermal images were used to compare the thermal variations associated to the bruxism, specifically when using the resilient occlusal device.

Special attention is given to an inspection performed with images in three different stages: before the experiments (at a resting interval), during the masticatory movements (i.e., when using of the device, according to task III), and 5 min after ending the movements. The analysis is carried out to verify the achievement of fatigue, as deduced from the FBG sensor results, obtaining another fatigue indicator from temperature changes in the muscles.

Fig. 8 shows three obtained images, where it is possible to inspect three different areas of the face, which are highlighted by the black lines (L1, L2 and L3) connecting a few specific anatomical points (marked by thermal targets attached to the skin face). The placement of the targets is dictated by the anatomy of diverse group of muscles involved in masticatory and facial movements. Line L1 follows the group of anterior and middle fibers of the temporal muscle, line L2 identifies the auricular region (masseter and medial pterygoid muscles) and line L3 marks the position of the masseter muscle. L2 and L3 lines are chosen also because they identify positions where the superficial muscle groupings are approximately equal. These lines are at the same temperature before masticatory activity, as can be observed in Fig. 8 (a). However, after the masticatory activity, we can observe different temperatures near to the auricular region (line L2), and to the masseter muscle (line L3) which are associated to a different internal muscular grouping (medial pterygoid muscle), as can be observed in Fig. 8 (b). From this preliminary analysis, it is noticed an increment in the overall facial temperature, indicated by the increase of temperature (most noticeable in the region close to L1) when exercising 
(Fig. 8(a) and 8(b)). After the end of the masticatory movements (Fig. 8(c)) the temperature gradually reduces, quantified by the mean value along each of the chosen lines.

The graph on Fig. 9 shows the average temperature evolution as a function of time for each line (L1, L2, L3). It is possible to notice that after 5 min (last cycle), the temperature goes back to the initial value. This investigation was prompted by previous studies that showed that the resilient material of the device induces the patient to perform a larger number of bite events during sleep and consequently greater muscle activity, which may cause muscle fatigue. As the temperature increases during the fatigue, changes in blood flow and consequently muscle injuries are among the causal factors of muscle fatigue.

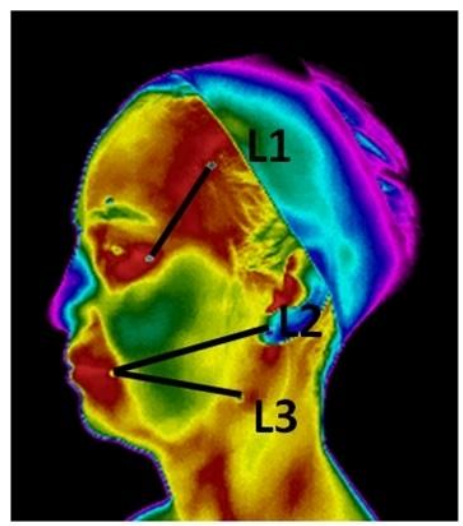

(a)

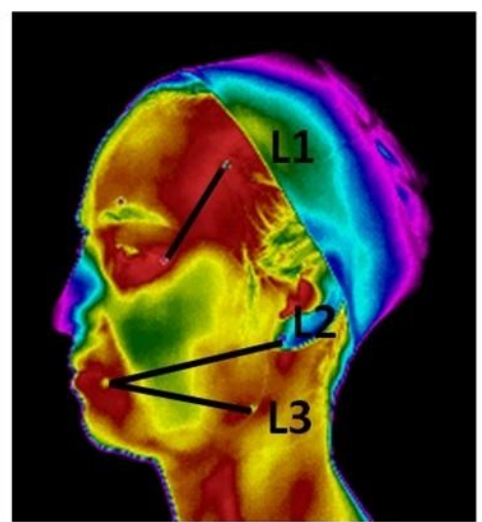

(b)

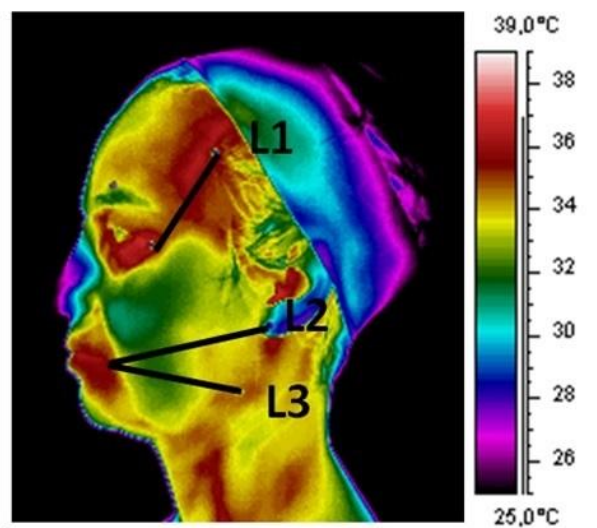

(c)

Fig. 8. Images showing the thermal changes: (a) before, (b) during (task IV), and (c) 5 min after the use of the occlusal device.

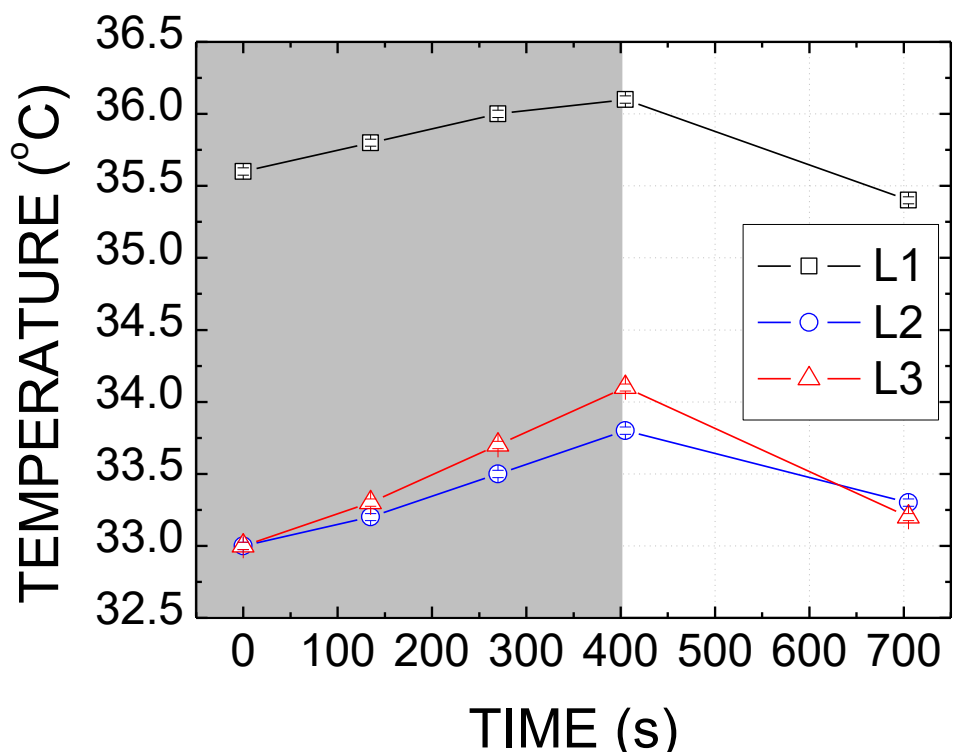

Fig. 9. Average temperature as a function of time along each selected region during the mastication procedure, the shaded area shows the exercising interval. (Symbols are the experimental data; the lines are only to guide the eyes). 
Previous studies showed the influence of fatigue on muscle temperature. Baldini et al. [33] reported the increments in facial temperature of $0.20^{\circ} \mathrm{C}$ by using the occlusal device in military pilots with bruxism, without the induced masticatory activity. In this case, they found out that the occlusal device increase the muscular temperature, which can be associated with muscle relaxation [33]. The increase of temperature can also be associated to the muscle activity. Bartuzi et al. reported the increase in the temperature during the fatigue tests in biceps [34]. In the results presented at our study, there was an increase of the temperature with the use of the occlusal device and with the induction of masticatory activity; the maximum temperature variation obtained was of $1.1^{\circ} \mathrm{C}$; so these results are in agreement with the literature.

\section{CONCLUSION}

In this case study, we demonstrated a new method in order to investigate the process of muscle fatigue in parafunctional patients using fiber Bragg gratings. The main advantage of using FBG inserted into resilient occlusal device is the possibility of obtaining force values in its own device, i.e. the one used for the treatment of bruxism symptoms. This allows forces analysis to be performed during patient treatment. The sensor characteristics show the feasibility of its use for dynamic analysis and acquisitions in situ and in real cases studies. In fact, the use of FBG sensors allows punctual forces measurements at the contact point of occlusion.

The sensor was evaluated in a case study with exhaustive muscular activity induced in a short period, in order to assess the muscle fatigue. The results obtained revealed that the sensor is a powerful tool for characterization of the muscular activity, including the identification of characteristic cycles, like exhaustion and point of fatigue. The understanding of these characteristics may help not only in the investigation, but also at the treatment of the symptoms of patients in the case of bruxism.

\section{ACKNOWLEDGMENT}

The authors acknowledge CAPES, CNPq, FUNDAÇÃO ARAUCARIA, FINEP, UTFPR and PUC-PR for scholarships and funding. Prof. Kalinowski thanks the Federal University of Technology - Paraná, for receiving him as a voluntary researcher during part of time used in this work.

\section{REFERENCES}

[1] J.P. Okeson, "Orofacial pain: guidelines for assessment, diagnosis, and management", Quintessence Publishing Co. Inc., Chicago, 1997.

[2] G.J. Lavigne, P.H. Rompre, J.Y. Montplaisir, "Sleep bruxism: Validity of clinical research diagnostic criteria in a controlled polysomno graphic study", Journal of Dental Research, vol. 75, pp. 546-52, 2016.

[3] S. Shetty, V. Pitti, B.C.L. Satish, G.P.S. Kumar, "Bruxism: A literature review", The Journal of Indian Prosthodontic Society, vol. 10 , pp. 141-148, 2010.

[4] A. De Laat, G.M. Macaluso, "Sleep bruxism as a motor disorder", Movement Disorders, vol. 17, 2002.

[5] G.L. Lavigne, P.H. Rompre, G. Poirier, H. Huard, T. Kato, J.Y. Montplaisir, "Rhythmic mastigatory muscle activity during sleep in humans", Journal of Dental Research, vol. 80, pp. 443-8, 2001.

[6] M.Z. Al-Ani, S.J. Davies, R.J.M. Gray, P. Sloan, A.M. Glenny, "Stabilisation device therapy for temporomandibular pain dysfunction syndrome", Cochrane Database Syst Rev, vol. 1, 2004. 
[7] G.M. Tartaglia, G. Lodetti, G. Paiva, C.M.D. Felicio, C. Sforza, "Surface electromyographic assessment of patients with long lasting temporomandibular joint disorder pain", Journal of Electromyography and Kinesiology, vol. 21, pp. 659-664, 2011.

[8] E.L. Schiffman, J.R. Fricton, D. Haley, "The relationship of occlusion, parafunctional habits and recents life events to mandibular dysfunction in a non-patient population", Journal of Oral Rehabilitation, vol. 19, pp. 201-223, 1992.

[9] Z.J. Liu, K. Yamagata, Y. Kasahara, G. Ito, "Electromyographic examination of jaw muscles in relation to symptoms and occlusion of patients with temporomandibular joint disorders", Journal of Oral Rehabilitation, vol. 26, pp. 33-47. 423, 1999.

[10] B.K. Benjamin, R.M. Enoka, "The neurobiology of muscle fatigue: 15 years later", Integrative and comparative biology, vol. 47, pp. 465-473, 2007

[11] H. Green, "Mechanism of muscle fatigue in intense exercise", Journal of sports sciences, vol. 15, pp. 247-256, 1997.

[12] D.G. Allen, J. Lannergren, H. Westerblad, "Muscle cell function during pro longed activity: cellular mechanisms of fatigue", Experimental physiology, vol. 80, pp. 497-527, 1995.

[13] B.A. Silva, F.G. Martinez, A.M. Pacheco, I. Pacheco, "Efeitos da fadiga muscular induzida por exercícios no tempo de reação muscular dos fibulares em indivíduos sadios", RevBrasMed Esporte, vol. 12, pp. 85-89, 2006.

[14] J. Williams, G. Klug, "Calcium exchange hypothesis of skeletal muscle fatigue: a brief review”, Muscle \& nerve, vol. 18, pp. 421-434, 1995.

[15] S.S. Yeung, A.L Au., C.C. Chow, "Effects of fatigue on the temporal neuro muscular control of vastus medialis muscle in humans", European journal of applied physiology and occupational physiology, vol. 80, pp. 379-385, 1999.

[16] R. Fitts, "Mechanisms of muscular fatigue", Principles of exercise biochemistry, Karger Publishers, Vol. 46, pp. 279-300, 2004.

[17] A. Adam, C.J.D. Luca, "Firing rates of motor units in human vastus lateralis muscle during fatiguing isometric contractions", Journal of Applied Physiology, vol. 99, pp. 268-280, 2005.

[18] M. Farella, K. Soneda, A. Vilmann, C.E. Thomsen, M. Bakke, "Jaw muscle soreness after tooth-clenching depends on force level", Journal of dental research, vol. 89, pp. 717-721, 2010.

[19] M. Bakke, C.E. Thomsen, A. Vilmann, K. Soneda, M. Farella, E. Moller, "Ultrasonographic assessment of the swelling of the human masseter muscle after static and dynamics activity", Archives of oral biology, vol. 41, pp. 133-140, 1996.

[20] A.A. Monteiro, S. Kopp, "The sufficiency of blood flow in human masseter muscle during endurance of biting in the intercuspal position and on a force transducer", Proceedings of the Finnish Dental Society, vol. 85, pp. 261-72, 1989

[21] S.K. Hunter, "Sex differences in human fatigability: mechanisms and insight to physiological responses", Acta Physiologica, vol. 210, pp. 768-789, 2014

[22] Y.J. Kim, T. Kudoki, Y. Tsukiyama, K. Koyano, G.T. Clark, "Haemodynamic changes in human masseter and temporalis muscles induced by different levels of isometric contraction", Archives of Oral Biology, vol. 44, pp. 641-50, 1999.

[23] H.J. Kalinowski, I. Abe, J.A. Santos, A. Ramos, "Application of fibre Bragg grating sensors in biomechanics", Trends in Photonics, Transworld Research Network, Trivandrum, India, 2010.

[24] I. Abe, M.S. Milczewski, M.A. Souza, H.J. Kalinowski, O.F. Machuca, G.C. Marin, E.S. Camargo, "The force magnitude of a human bite measured at the molar intercuspidation using fiber Bragg gratings", Journal of Microwaves, Optoelectronics and Electromagnetic Applications, vol. 16, pp. 434-444, 2017.

[25] A.P.G.O. Franco, A.O. Gebert, M.A. Souza, L. Jeranoski, H.J. Kalinowski, I. Abe, "Measurement of mandibular movements in parafunctional patient using occlusal device with Bragg gratings: pilot study", Sixth European Workshop on Optical Fibre Sensors, International Society for Optics and Photonics, Vol. 9916, 2016.

[26] M.S. Milczewski, J.C.C. Silva, I. Abe, A.S. Paterno, J.A. Simões, H.J. Kalinowski, "Measuring orthodontic forces with HiBi FBG sensors", Proceedings of 18th Optical Fibre Sensors Conference, Cancun, pp. 23-27, 2006.

[27] M.S. Milczewski, J.C.C. da Silva, C. Martelli, L. Grabarski, I. Abe, H.J. Kalinowski, "Force monitoring in a maxilla model and dentition using optical fiber Bragg gratings", Sensors, vol. 12, pp. 11957-65, 2012a.

[28] M.S. Milczewski, M. Stevenson, J. Canning, C. Mattelli, H.J. Kalinowski, "Sensitivity of silica and polymer microstructured fibres transversal pressure", Glass Technology-European Journal of Glass Science and Technology Part A, vol. 50, pp. 211-213, 2012b.

[29] S.C. Tjin, Y.K. Tan, M. Yow, J. Hao, "Recording compliance of dental device use in obstructive sleep apnea patients by force and temperature modelling", Medical and Biological Engineering and Computing, vol. 39, pp. 182-184, 2001.

[30] G. Werhle, P. Nohama, H.J. Kalinowski, P.L. Torres, L.C.G. Valente, "A fibre optic Bragg grating strain sensor for monitoring ventilator movements", Measurement Science and Technology, vol. 12, pp. 805-809, 2001.

[31] S. Umesh, S. Padma, S. Asokan, T. Srinivas, "Fiber Bragg grating based bite force measurement", Journal of biomechanics, vol. 49, pp. 2877-2881, 2016.

[32] J.S. Heo, J.H. Chung, J.J. Lee, "Tactile sensor arrays using fiber Bragg grating sensors", Sensors and Actuators A: Physical, vol. 126, pp. 312-327, 2006.

[33] A. Baldini, A. Nota, C. Cioffi, F. Ballanti, P. Cozza, "Infrared thermographic analysis of cranio facial muscles in military pilots affected by bruxism", Aerospace medicine and human performance, vol. 86, pp. 374-378, 2015.

[34] P. Bartuzi, D. Roman-Liu, T. Wisniewski, "The influence of fatigue on muscle temperature", International Journal of Occupational Safety and Ergonomics, vol. 18, pp. 233-243, 2012. 